\title{
Research Paper \\ Estimating the relationship between the output gap and inflation in Iraq for the period 1989-2018
}

Journal of

TANMIYAT ALRAFIDAIN

\section{(TANRA)}

A scientific, quarterly, international, open access, and peer-reviewed journal

Vol. 40, No. 130

June 2021

(C) University of Mosul |

College of Administration and Economics, Mosul, Iraq.

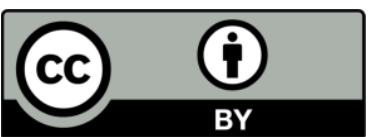

TANRA retains the copyright of published articles, which is released under a "Creative Commons Attribution License for CC-BY-4.0" enabling the unrestricted use, distribution, and reproduction of an article in any medium, provided that the original work is properly cited.

Citation:?, Abdulrahman. M , AlJwejatee, Aws F. A (2021). "Estimating the relationship between the output gap and inflation in Iraq for the period 1989-2018". TANMIYAT ALRAFIDAIN, 40 (130), 1 To 100 ,

https://doi.org/ 10.33899/tanra.2020.128019.10 42

P-ISSN: 1609-591X e-ISSN: 2664-276X tanmiyat.mosuljournals.com

\author{
Abdulrahman M. A ${ }^{1}$., Aws F. A. Al- Jwejatee ${ }^{1}$ \\ ${ }^{1 \& 2}$ College of Administration and economics/ Mosul University
}

Corresponding author: Abdulrahman Mohammed A.

. abdulrahmanakrawi85@gmail.com

DOI: https://doi.org/ 10.33899/tanra.2020.128019.1042

Article History: Received: 15/8/2020; Revised: 12/9/2020; Accepted: 22/9/2020; Published: 1/6/2021.

\section{Abstract}

The research presents how to estimate the relationship between the output gap and inflation in the Iraqi economy, by estimating the output gap using the Hodrick-Prescott filter on GDP data in Iraq for the period 19892018 and finding the changes in the output gap as a result of changes in GDP as a result of conditions Numerous, then review the data on inflation rates in Iraq for the same period and note the major developments that accompanied the change in the rate of inflation, and then find the relationship between the output gap as an independent variable that affects the rate of inflation as a variable dependent on changes in the total output. The relationship between the variables was estimated using the selfregression model of the slowed distributed time slots (ARDL), and the form of the relationship between the variables was reached where there is a long-term balance relationship between the changes that occur in the output gap and its effect on inflation rates.

\section{Keywords}

GDP Gap, Inflation Rate, Hodrick-Prescott filter, ARDL Model 


\section{ورقة بحثية \\ ثقدير العلاقة بين فجوة الناتج والتضخم في العراق للمدة

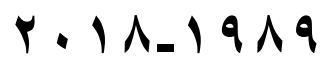

المؤلف المراسل: عبدالرحمن محمد أحمد عقراوي، جامعة الموصل، كلية الإدارة والاقتصاد، abdulrahmanakrawi85@gmail.com.

DOI: https://doi.org/ 10.33899/tanra.2020.128019.1042

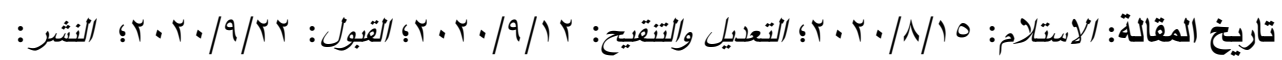

$$
\cdot r \cdot r / T / 1
$$

المستخلص

يسعى البحث إلى الكثف عن كيفية تقدير العلاقة بين فجوة الناتج والتضخم في الاقتصاد العراقي،

وذلك من خلال تقدير فجوة الناتج باستخدام مشح هودريك- بريكوت على بيانات الناتج المحلي الإجمالي في العراق للفترة 9119 111 •r وإيجاد التغيرات الحاصلة في فجوة الناتج نتيجة للتغيرات الحاصلة في الناتج المحلي الإجمالي نتيجة لظروف عديدة، ومن ثم استعراض البيانات الذاصة بمعدلات التضخم في العراق للفترة نغسها وماحظة التطورات الكبيرة التي رافقت التغير في معدل التضخم، ومن ثم إيجاد العلاقة بين فجوة الناتج كمتغير مستقل يؤثر على معدل التضخم كمتغير تابع للتغيرات التي تحصل في الناتج الإجمالي. وتم تقدير العلاقة بين المتغيرات استخدام أندوذج الانحدار الذاتي للفجوات الزهنية الموزعة المتباطئة (ARDL)، وتم التوصل إلى شكل العلاقة بين المتغيرات حيث يوجد هناك علاقة توازنية طويلة الاجل بين التغيرات التي تحصل في فجوة الناتج وتأثيرها على معدلات التضخم.

الكملمات المفتاحية

فجوة الناتج، معدل التضخم، مرشتح هودريك- بريسكوت، أنموذج (ARDL)

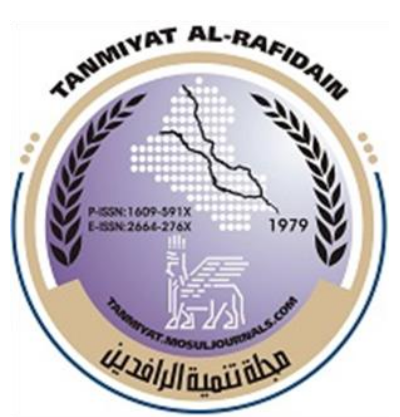

هبلة

\section{تنسمبية الرافدين}

(TANRA) دولية، مفتوحة الوصول، محكمة.

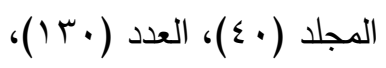

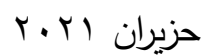

(C) جامعة الموصل ج كلية الإدارة والاقتصاد، الدوصل، العراق.

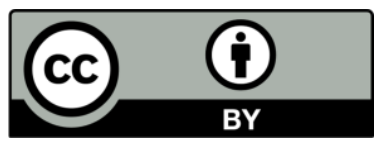

تحتفظ (TANRA) بحقوق الطبع والنشر للمقالات المنشورة، والتي يتم إصدارها بموجب ترخيص

\lrcorner (Creative Commons Attribution) (CC-BY-4.0)

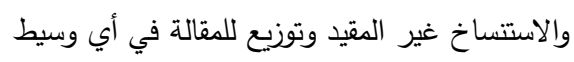
نقل، بشرط اقتباس العمل الأصلي بشكل صحيح.

الاقتباس: عقراوي، عبدالرحمن محمد

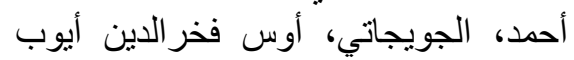

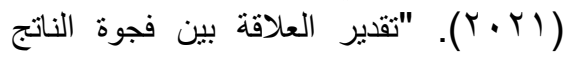

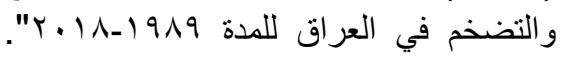

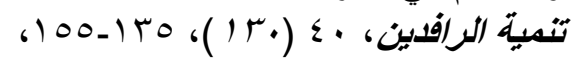

https://doi.org/

10.33899/tanra.2020.128019.10 42

P-ISSN: 1609-591X

e-ISSN: 2664-276X

tanmiyat.mosuljournals.com 
تعتبر عملية استقرار المستوى العام للأسعار من أهم الأولويات التي تسعى إليها السياسة

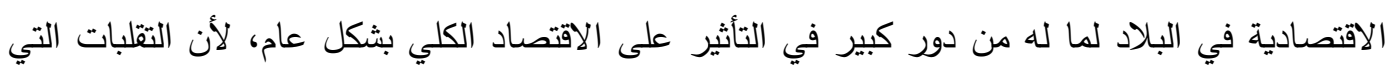

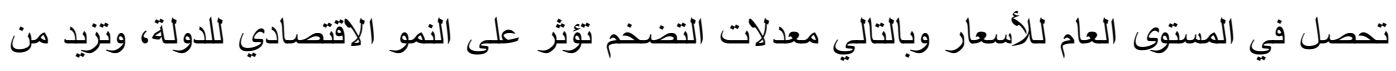

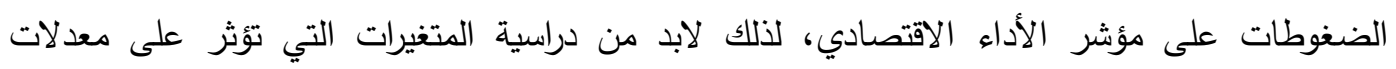

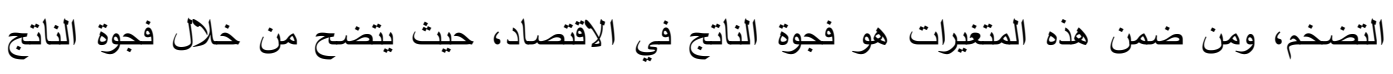

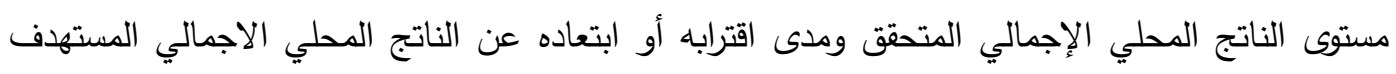
الذي من خلاله يتم الحفاظ على مستويات الأسعار ومعدلات التضخئ.

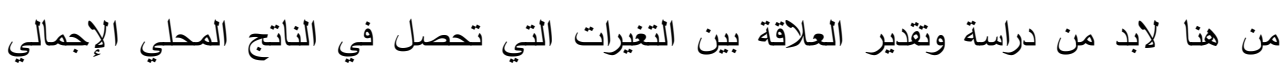
المتحقق والناتج المحلي الإجمالي المستهدف من جهة والتغيرات التي تحصل في معدلات التضخم من جهة

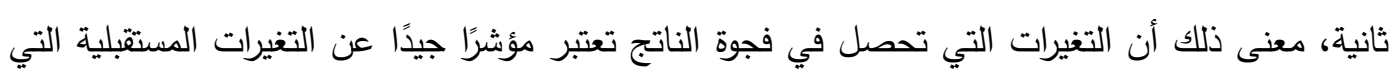

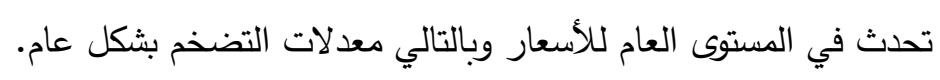
أهمية البحث

تكمن أهمية البحث في كيفية تقدير العلاقة بين فجوة الناتج وفقاً للتغيرات التي تحصل فيها نتيجة

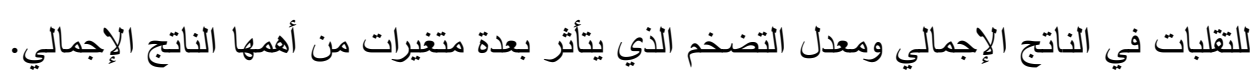
مشكلة البحث

إن المشكلة التي تواجه البحث هي في كيفية تقدير فجوة الناتج من خلال احتساب الناتج المستهدف ومدى انحرافه عن الناتج المتحقق، وكذلك في طبيعة تأثير التغيرات في فجوة الناتج على معدل التضخد نتيجة للتغيرات في الطلب الكلي الفعال. هدف البحث

يهدف البحث إلى إيجاد الطريقة المثلى في احتساب الناتج المستهدف، وبالتالي فجوة الناتج من جهة كمتغير مستقل يؤثر بالنتيجة على معدل التضخم كمتغير تابع. فرضية البحث

يفترض البحث وجود علاقة توازنية قصيرة الأجل وطويلة الأجل بين فجوة الناتج ومعدل التضخم، وهذا ما سيتم إثبات صحته من خلال التحليل القياسي للعلاقة بين المتغيرين. منهجية البحث من منيت يستند البحث إلى منهجين في التحليل، الأول منهج نظري يتم من خلاله توضيح مفهوم فجوة الناتج وكيفية احتسابها، فضلاً عن تحليل الفجوة في العراق الخاصة بمدة الدراسة، إضافة إلى توضيح مفهوم

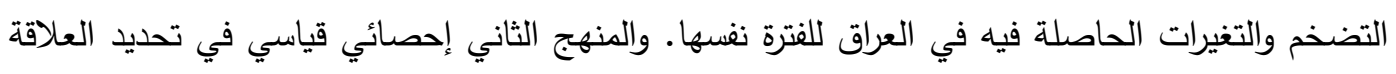
بين فجوة الناتج ومعدل التضخم في العراق وإيجاد شكل العلاقة بين متغيري البحث. 


\section{I - فجوة الناتج GDP Gap}

فجوة الناتج هي الفرق بين الناتج الفعلي (Actual GDP) والناتج المتوقع انتاجه ( Potential)

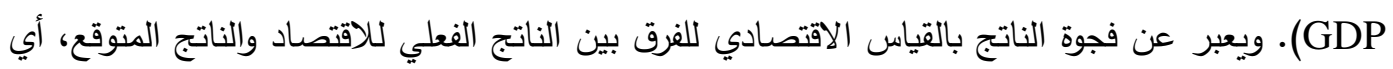

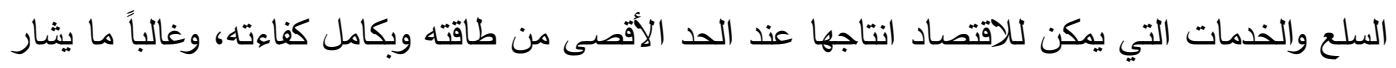

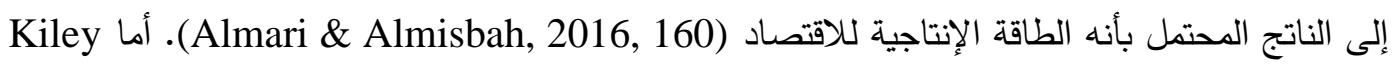
فيعطي ثلاثة تعريفات لفجوة الناتج، الأول هو مدى ابتعاد الناتج عن اتجاهه العشوائي على المدى الطويل; والثاني انحراف الناتج عن المستوى المتكافئ مع التكنولوجيات الحالية والاستخدام العادي لرأس المات المال والتوظيف; والثالث هو مدى انحراف الناتج عن المعدل الطبيعي له (Kiley, 2013,1).

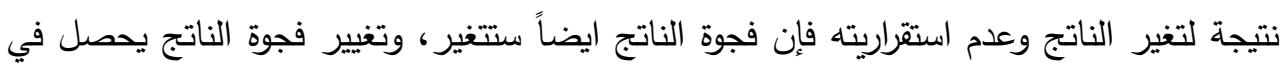
اتجاهين: الاتجاه الموجب والاتجاه السالب، وكلا الاتجاهين غير جيدين بالنسبة للاقتصاد، إذ يعد الاستقرار الاقتصادي من الأهداف الرئيسة لواضعي السياسات الاقتصادية، حيث تحدث الفجوة الموجبة عندما يكون الاهن

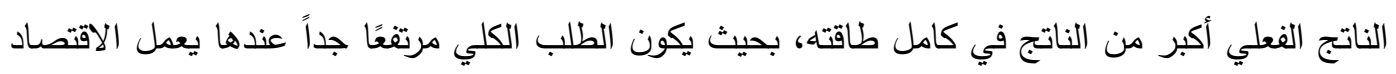

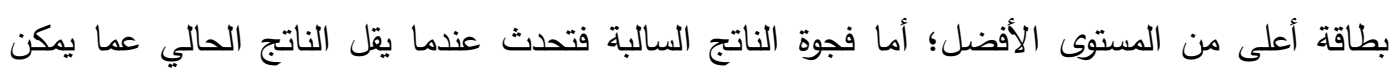

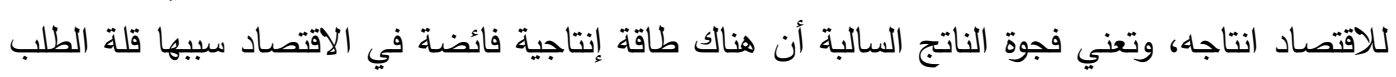

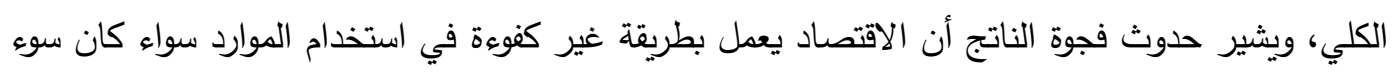
الاستخدام متمثلا بالهدر أو استغلال الموارد عن الحد الطبيعي (Jahan \& Mahmood, 2013, 38).

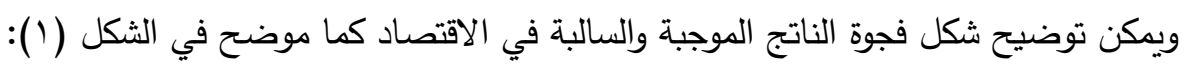

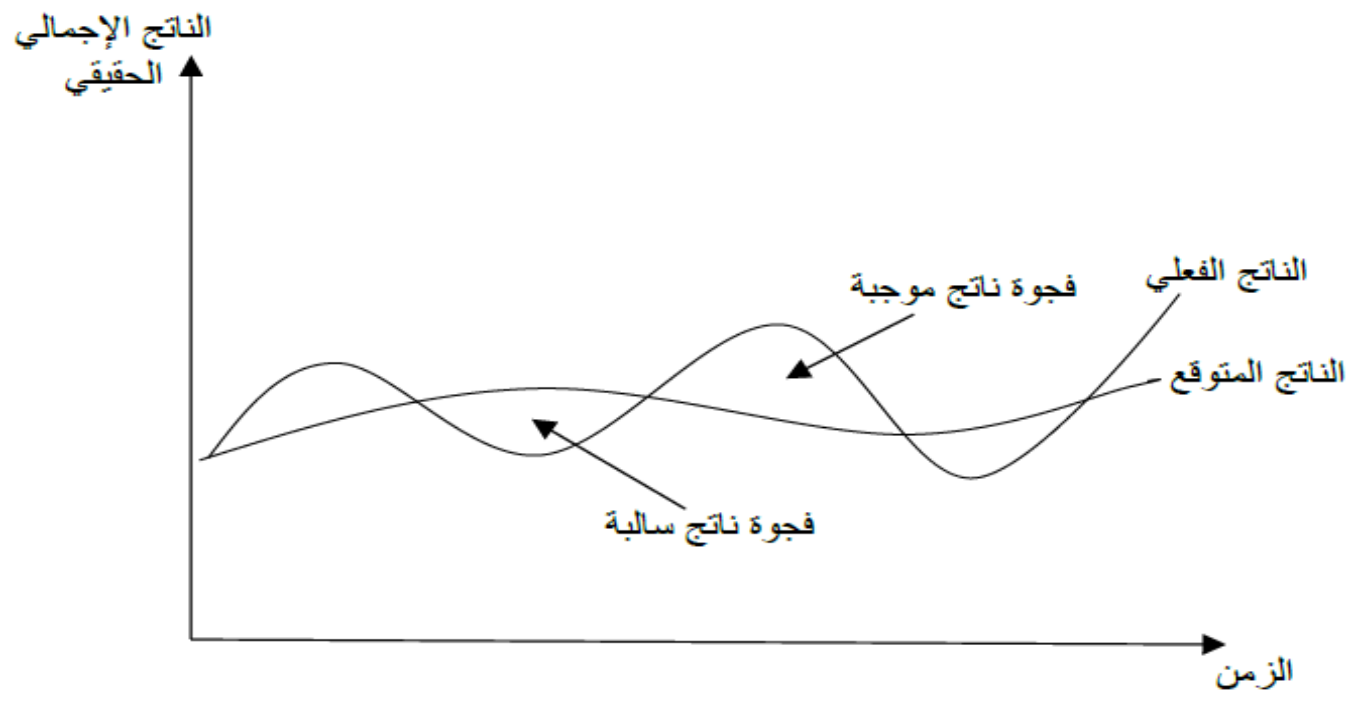

الثكل ا: فيوة الناتج الموجبة والسالبة

Source: Apel, Mikael. Hansen, Jan. Lindberg, Hans. (1996), Potential output and output gap, Sverige's Riksbank, Quarterly Review, P. 26 
يوضح الثكل السابق فجوة الناتج الموجبة والسالبة، إذ يهتم واضعو السياسات الاقتصادية في تقدير

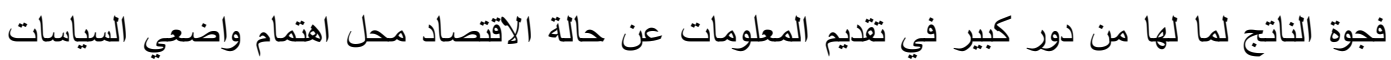
في بناء سياساتهم النقدية الهادفة إلى تقديم الحلول والمعالجات للتقلبات الاقتصادية الدورية وغير الدورية التي تترك أثرها السلبي على الاقتصاد (Almari \& Almisbah, 2016, 160).

Multivariate Kalman Filter 1- 1 مرشح كالمن متعدد المتغيرات يعتبر هذا المرشح أو الطريقة امتدادًا لمرشح كالمن وحيد المتغيرات، لكنه يتضمن معادلات إضافية معتمدة على العلاقات الاقتصادية، إذ أضاف بعض الاقتصاديين العلاقات التالية مثلا إن الناتج المحتمل Kramer \& ) يفترض اتباع السير العشوائي، أو إن التضخم يترابط مع التضخم الحاصل في الماضي العني Farrell, 2014, 13

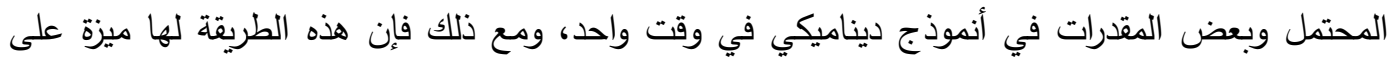

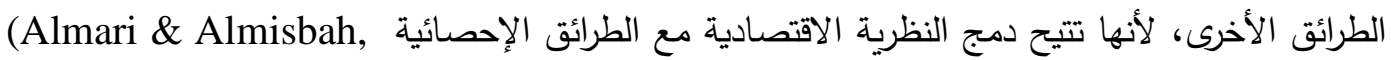
.2016, 163)

1-r حساب فجوة الناتج وتقديرها في الاقتصاد العراقي يمكن تقدير فجوة الناتج في الاقتصاد العراقي بالاعتماد على بيانات الناتج الفعلي الصادرة عن العادي البنك المركزي العراقي، والبيانات الناتجة عن تقدير الناتج المتوقع بالاعتماد على مرشح كالمن متعدد بلاتدات

$$
\text { المتغيرات MKF وكما في الجدول (1): }
$$

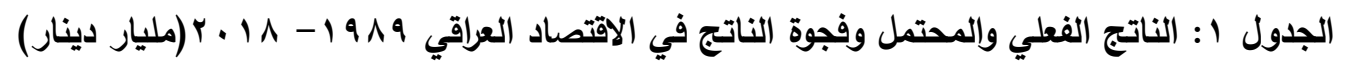
(بدون القطاع النفطي)

\begin{tabular}{|c|c|c|c|c|c|c|c|}
\hline $\begin{array}{c}\text { فجوة الناتج } \\
\text { (r) } \\
3=1-2\end{array}$ & المتوقع (r) & الناتج الفعلي & السنة & $\begin{array}{c}\text { فجوة الناتج } \\
\left(\begin{array}{r}(\mu \\
3=1-2\end{array}\right.\end{array}$ & المتاتج & الناتج الفعلي & السنوات \\
\hline 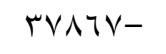 & $097 \wedge 0, \Sigma$ & $Y \backslash \wedge) \wedge, \Sigma$ & $r \ldots \varepsilon$ & 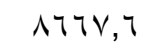 & $1 \leqslant 07 ., 0$ & rrrrA, & 1919 \\
\hline rotrr- & $T \cdot V \leqslant 1, r$ & $r 0119, r$ & $r \ldots o$ & $r \cdot q v, 0$ & I. r०ד, r & ג וror & 199. \\
\hline - & $\neg) \wedge \vee, \Lambda$ & rAOrr, $q$ & $r \ldots q$ & 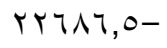 & $\Gamma \cdot \varepsilon \diamond \wedge, \varepsilon$ & $\vee \vee \vee 1, q$ & 1991 \\
\hline - & TrAOV,V & rVVTr,I & $r \ldots v$ & $19171, r-$ & rYAOY, Y & $\mid r \longleftarrow \wedge \varepsilon, r$ & 1994 \\
\hline$r \wedge 01 Y-$ & $77 \wedge 07,9$ & $r \wedge T \leqslant \varepsilon, q$ & $r \ldots \Lambda$ & $1 \wedge \leqslant \vee r, O-$ & $r \Psi \leqslant \vee \wedge, \vee$ & $10.0, r$ & 1994 \\
\hline rฯฯVА, Y- & TVOY & $r \cdot \wedge \leq r, l$ & $r . . q$ & $1 \wedge \mid \vee 0,1-$ & พч人97, & $|\wedge \vee Y|, \Lambda$ & 1998 \\
\hline r. & $\vee \cdot \leqslant ० \wedge, \wedge$ & $r \leqslant r q 7, r$ & $r .1$. & YrTr,A- & rчqАV,० & $1 \leqslant 70 \leqslant, V$ & 1990 \\
\hline$r \leqslant \leqslant 99,9-$ & $V) \leq 0 \wedge, r$ & r & $r .11$ & -rד, & r $9 \leq 07, r$ & 17 1 & 1997 \\
\hline
\end{tabular}




\begin{tabular}{|c|c|c|c|c|c|c|c|}
\hline $91 \leq 1,1-$ & $V \leqslant 701,7$ & $7001 \cdot, 0$ & $r \cdot 1 r$ & rฯArV,৭- & $\varepsilon . O Y \varepsilon, Y$ & ז & $199 \mathrm{~V}$ \\
\hline r०97,r- & VVOTA,O & $v \leq q 9 \cdot, r$ & $r .1 r$ & -r, & $\leqslant 07 \wedge 9, \mathrm{~V}$ & $1 \leqslant O \vee \wedge, \varepsilon$ & 1991 \\
\hline$\Sigma \cdot Y \wedge, 0$ & A.VAr,r & $\Lambda \varepsilon \wedge 1, \wedge$ & $r .1 \leq$ & $r \leqslant r q \vee, q-$ & $0 . \vee 0 \wedge, 0$ & $17 \leq 7 \cdot, 7$ & 1999 \\
\hline $1 \cdot \wedge \wedge \wedge, \wedge-$ & NotrV,V & $\vee \leqslant \vee r \wedge, q$ & $r+10$ & 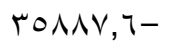 & Orrฯ人, & $17 \leqslant \wedge 1,1$ & $r \ldots$ \\
\hline$r \cdot \Lambda \cdot, r$ & VTror, & $\vee \wedge \Sigma \Psi Y, \wedge$ & $r .17$ & rVI Yo,V- & $0 \leq \vee \wedge 0,1$ & $1 \vee 709, \varepsilon$ & $r . .1$ \\
\hline $109 r, r$ & 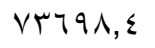 & vora., T & $r+1 v$ & 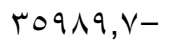 & $0 \leqslant Y M 0, T$ & $1 \wedge r \leqslant 0,9$ & $r \ldots r$ \\
\hline |Yฯ & $V 7107,9$ & $v \leqslant \wedge q,, r$ & $r \cdot \mid \Lambda$ & \&rVAr,r- & 07107,7 & r, & $r \ldots r$ \\
\hline
\end{tabular}

المصدر: من عمل الباحثين بالاعتماد على، العمود (1) وزارة التخطيط، الجهاز المركزي للإحصاء، مختلفة. العمود (r) من عمل الباحثين بالاعتماد على البرنامج الاحصائي EViews. العمود (r) من عمل الباحثين

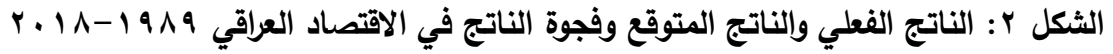

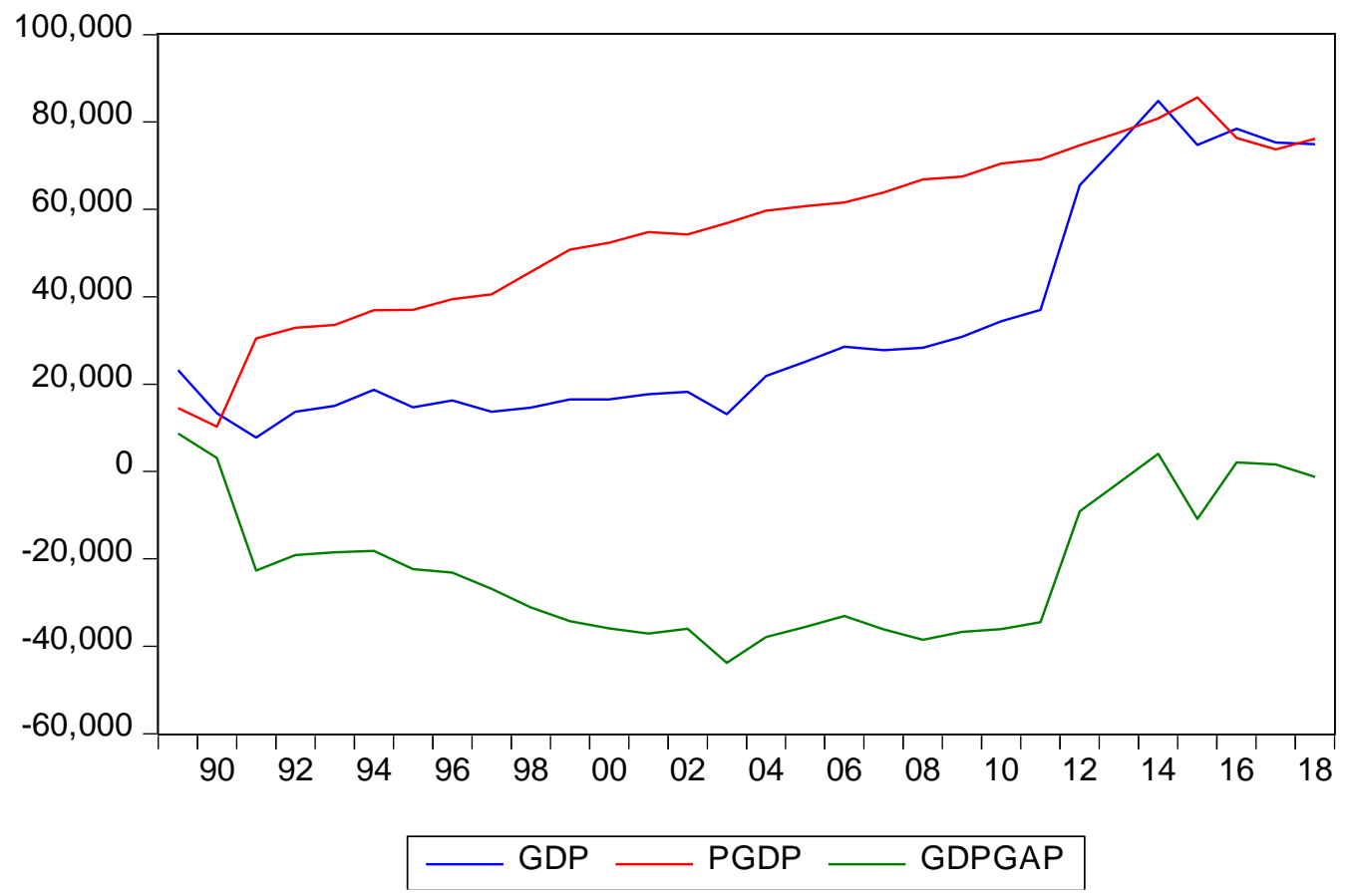

المصدر: من عمل الباحثين بالاعتماد على الجدول (1)

يتضح من الجدول (1) والثكل (Y) بأن نشاط الاقتصاد الكلي العراقي شهد انتعاشًا في عامي

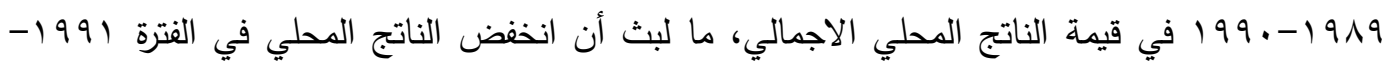

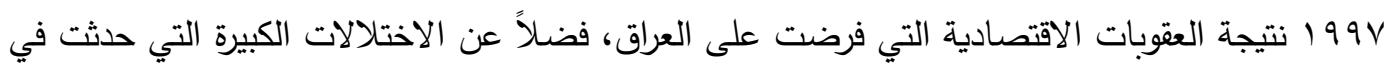




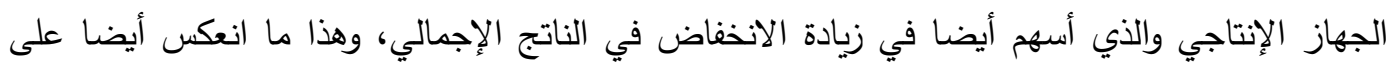

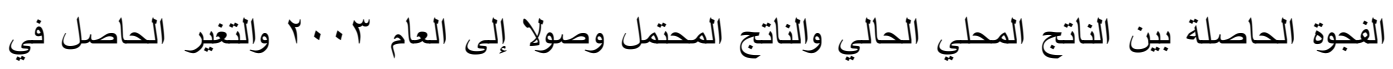
الاقتصاد العراقي، واستمر الناتج الفعلي في الانخفاض مقارنة مع الناتج المحتمل، مما زاد من فجوة الناتج

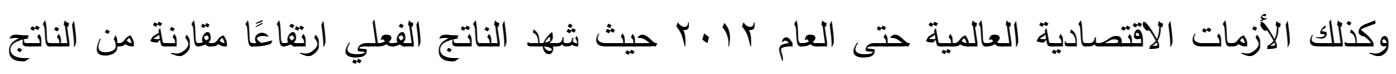
المحتمل، واستمر في ذلك مع فترات انخفاض متقطعة لأسباب اقتصادية وسياسية.

\section{rnflation Rate r معدل التضخم وسنر}

التضخم هو الارتفاع المستمر في المستوى العام للأسعار خلال مدة زمنية معينة، وعادة ما تكون

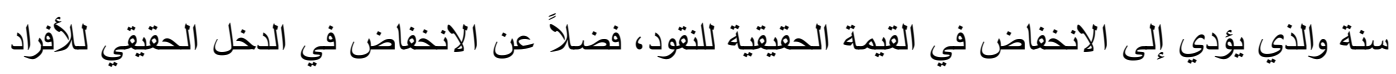

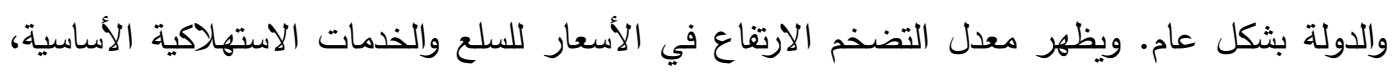

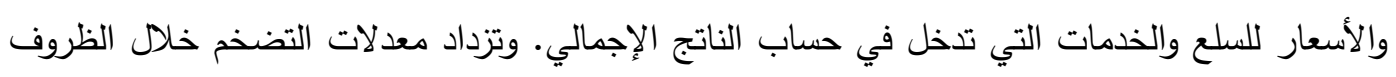

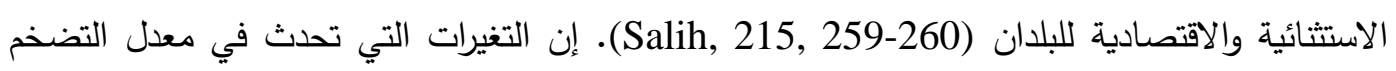
تعود غالباً إلى زيادة المعروض النقاتصادي من قبل البنوك المركزية لاسيما عندما لا يضاهي الطلب الكلي

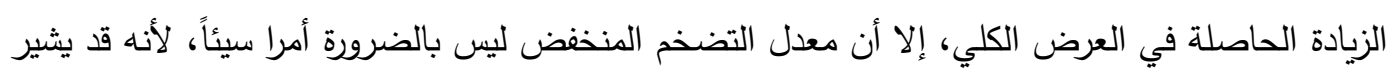
إلى حصول زيادة في النمو الاقتصادي (Szulczyk, 2014, 12). يرتبط مفهوم التضخم وفق آراء المدرسة الكينزية والمدرسة النقودية بشكل أساسي في التغيرات

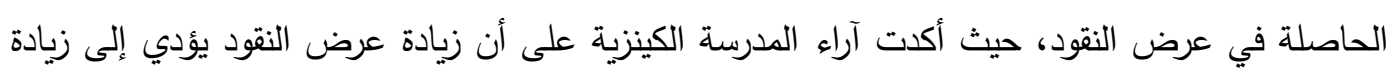

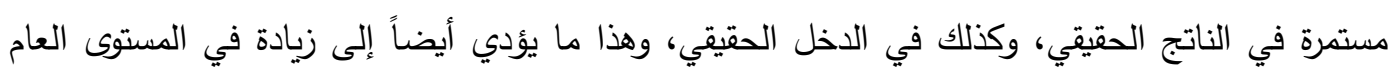

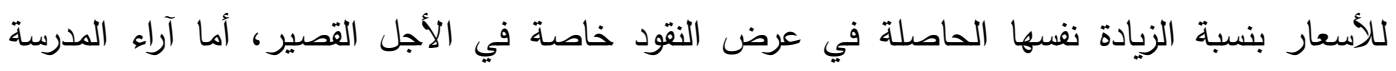

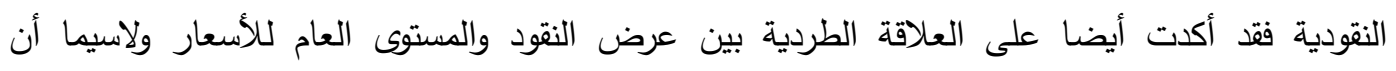

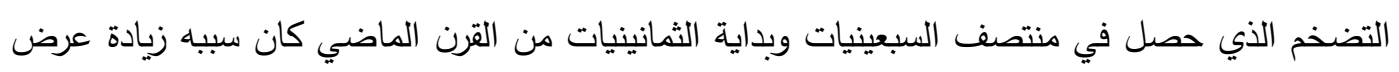

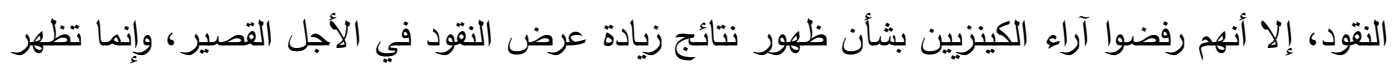
في الأجل الطويل، ذلك لأن سرعة تداول النقود تتأثر في الأجل الطويل نتيجة التطورات الحديثة في العمليات المصرفية (Najmaldin \& et, 2012, 346-347).

$$
\text { r - } 1 \text { تحليل معدل التضخم في العراق }
$$

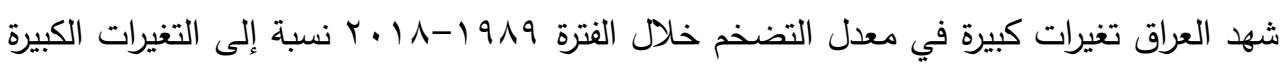

في الاقتصاد بشكل عام، وهو ما يتضح من خلال الجدول (r): 


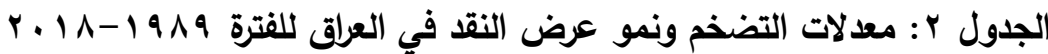

\begin{tabular}{|c|c|c|c|c|c|}
\hline معدل نمو عرض & التضخم معدل & السنة & معدل نمو عرض & التضخم معدل & السنة \\
\hline ro., 1 & $r q, q$ & $r \cdot \varepsilon$ & $r \cdot, 0$ & I, & 1919 \\
\hline Ir, & ru, & $r . . Q$ & $r q, \varepsilon$ & 01,7 & 199. \\
\hline ro, & or, & $r . . q$ & $7 \cdot, 7$ & $1 \wedge 7,0$ & 1991 \\
\hline$\varepsilon \cdot, \varepsilon$ & $r \cdot, v$ & $r \cdot v$ & $\vee V, q$ & $\Delta r, v$ & 1994 \\
\hline$r q, V$ & $r, V$ & $r . \Lambda$ & 97,1 & $r \cdot V, T$ & 1994 \\
\hline$r, r$ & $r, \Lambda-$ & $r . .9$ & $1 \vee 7,1$ & $\varepsilon 9 r$ & 1998 \\
\hline rی, , & $r, 0$ & $r \cdot 1$. & 190,1 & r, & 1990 \\
\hline$r \cdot, V$ & 0,7 & $r .11$ & ru, r & $10, \varepsilon$ & 1997 \\
\hline r & 7,1 & $r .1 r$ & $\Lambda$ & rr & $199 V$ \\
\hline 10,1 & $r, \varepsilon$ & $r \cdot 1 r$ & $r \cdot, r$ & $1 \varepsilon, V$ & 1991 \\
\hline $1,0-$ & $r, r$ & $r \cdot 1 \varepsilon$ & $q, v$ & $1 T, 0$ & 1999 \\
\hline $1 \cdot-$ & $1, \varepsilon$ & $r .10$ & $17, \varepsilon$ & $\varepsilon, 9$ & $r \ldots$ \\
\hline$\Lambda$ & 1,0 & Y.17 & ro & r, & $r . .1$ \\
\hline 1 & $\cdot, 0$ & $r .1 \mathrm{~V}$ & $r q, 0$ & $19, r$ & $r \ldots r$ \\
\hline $1, r$ & $\cdot, \varepsilon$ & $Y .11$ & 91,0 & r & $r \ldots r$ \\
\hline
\end{tabular}

المصدر: من إعداد الباحثين بالاعتماد على عمود (1) وزارة التخطيط/ الجهاز المركزي للإحصاء/ أباء بغداد، نشرات مختلفة، عمود (Y) البنك المركزي العراقي، نشرات مختلفة.

يتضح من الجدول (Y) أن هنالك توسعاً كبيراً في عرض النقد خلال الفترة 9199 19901، إذ إرتفع

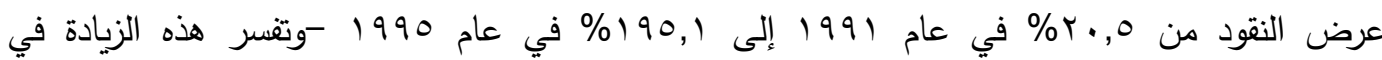
معدلات عرض النقد نتيجة الإصدار النقدي الجديد الذي قام به البنك المركزي العراقي لغرض تمويل

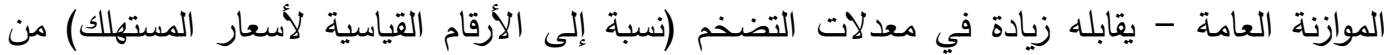

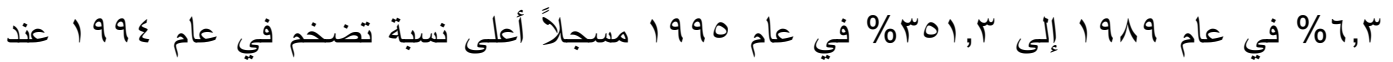

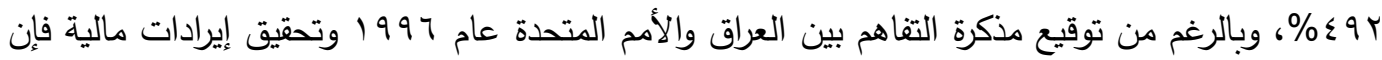

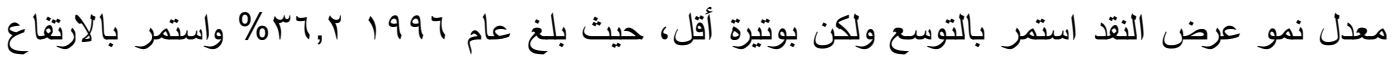




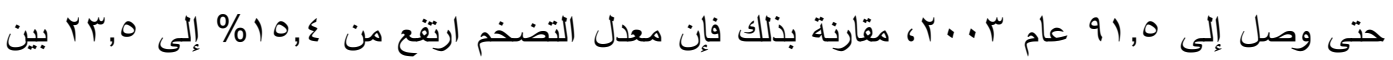

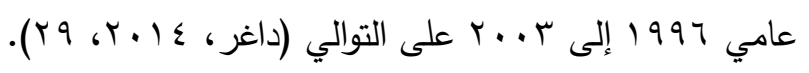

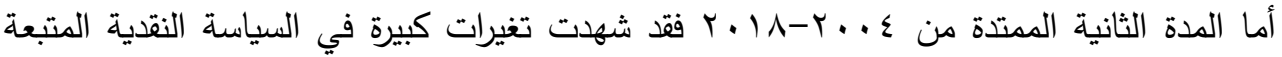

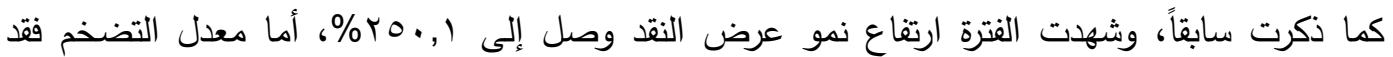

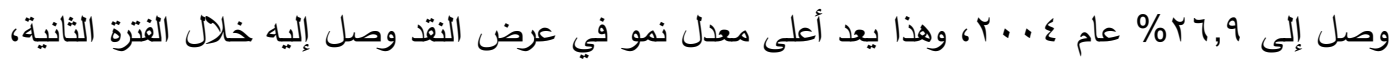

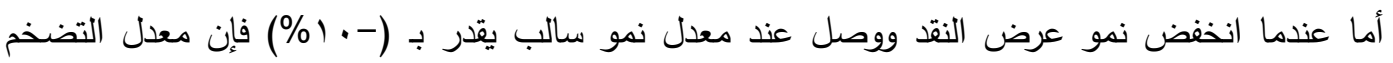

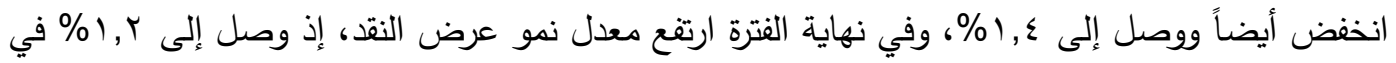

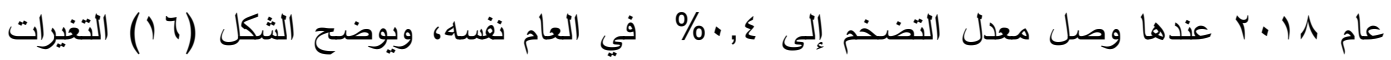
الحاصلة في كل من معدل التضخم ومعدل نمو عرض النقد (Kadhim \& et, 2019, 121).

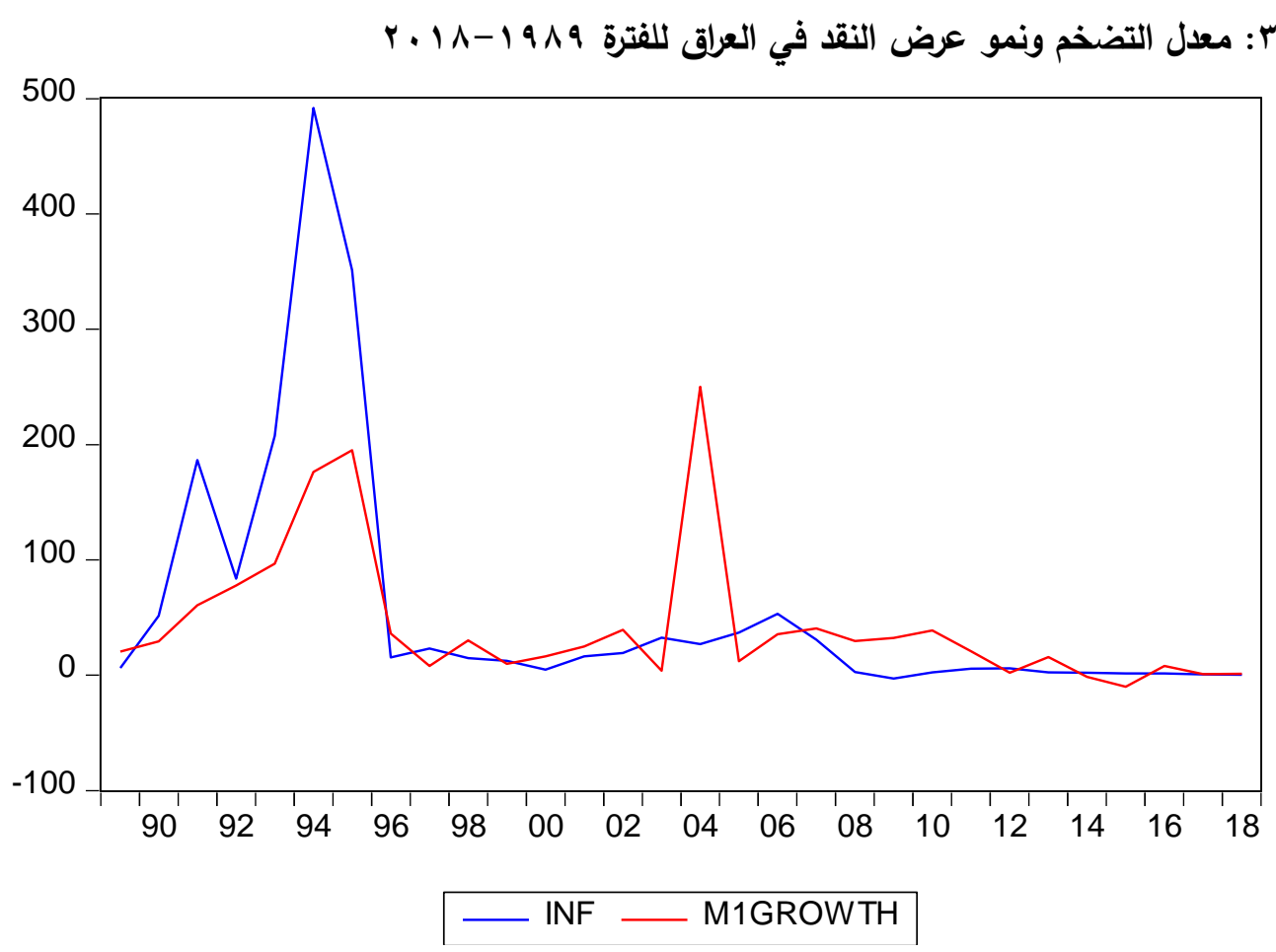

المصدر : من إعداد الباحثين بالاعتماد على بيانات الجدول (؟).

r- تقدير العلاقة بين فجوة الناتج والتضخم من أجل تقدير العلاقة بين فجوة الناتج والتضخم في العراق، لابد من استخدام الطرائق القياسية في تقدير العلاقة طويلة الأجل وقصيرة الأجل بين المتغيرات، ولغرض الوصول إلى الأنموذج القياسي الصحيح

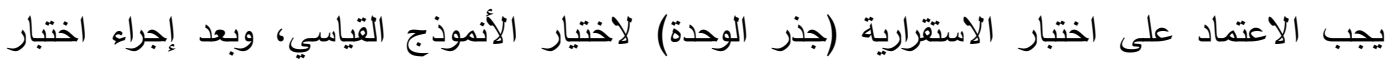


الاستقرارية تبين أن المتغير التابع فجوة الناتج والمتغير المستقل معدل التضخ متكاملان من الدرجة (0)، التهات مما يمكن الاعتماد على اختبار التكامل المشترك باستخدام أنموذج الانحدار الذاتي للفجوات الزمنية الموزعة فئنة المتباطئة (ARDL) في تقدير العلاقة بين المتغيرات وكما يتضح في الآتي. التئي.

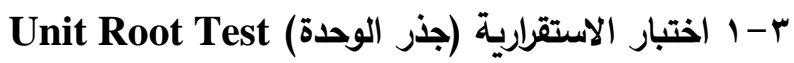
لغرض إجراء اختبار الاستقرارية (جذر الوحدة) على متغيرات الأنموذج، سيتم الاعتماد على اختبار

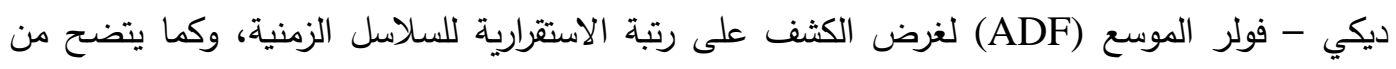

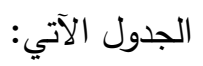

الجدول بّ: نتائج اختبار الاستقرارية (جذر الوحدة) لمتغيرات الأنموذج

\begin{tabular}{c|ccc}
\hline \multirow{2}{*}{ Variables } & \multicolumn{3}{|c}{ Augmented Dickey-Fuller Test (ADF) } \\
\cline { 2 - 4 } & \multicolumn{3}{|c}{ At Level } \\
\cline { 2 - 4 } & (ADF) Statistic & $\mathbf{5 \%}$ & $\mathbf{1 0 \%}$ \\
\hline INFLATION & -5.354766 & -3.574244 & -3.221728 \\
\hline GDP GAP & -3.986642 & -3.580623 & -3.225334 \\
\hline
\end{tabular}

EViews 10 المصدر: من إعداد الباحثين باستخدام برنامج

إذ يتضح من الجدول (ץ) أن المتغير التابع فجوة الناتج مستقر عند المستوى Level وعند القيم

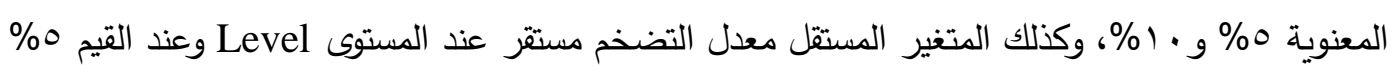

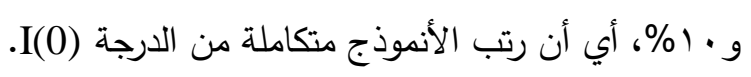

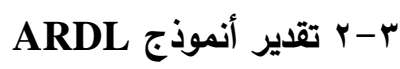
يتم من خلال تقدير أنموذج ARDL التعرف على قيم معامل R2 وكذلك التعرف على قيم

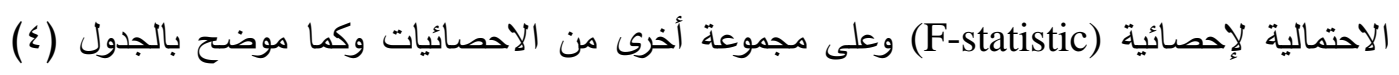
الآتي. 
Dependent Variable: INF

الجدول ؛ : تقدير أنموذج ARDL

Method: ARDL

Date: 08/03/20 Time: 14:10

Sample (adjusted): 19932018

Included observations: 26 after adjustments

Maximum dependent lags: 4 (Automatic selection)

Model selection method: Akaike info criterion (AIC)

Dynamic regressors (4 lags, automatic): GDP

Fixed regressors: $\mathrm{C}$

Number of models evalulated: 20

Selected Model: ARDL $(4,0)$

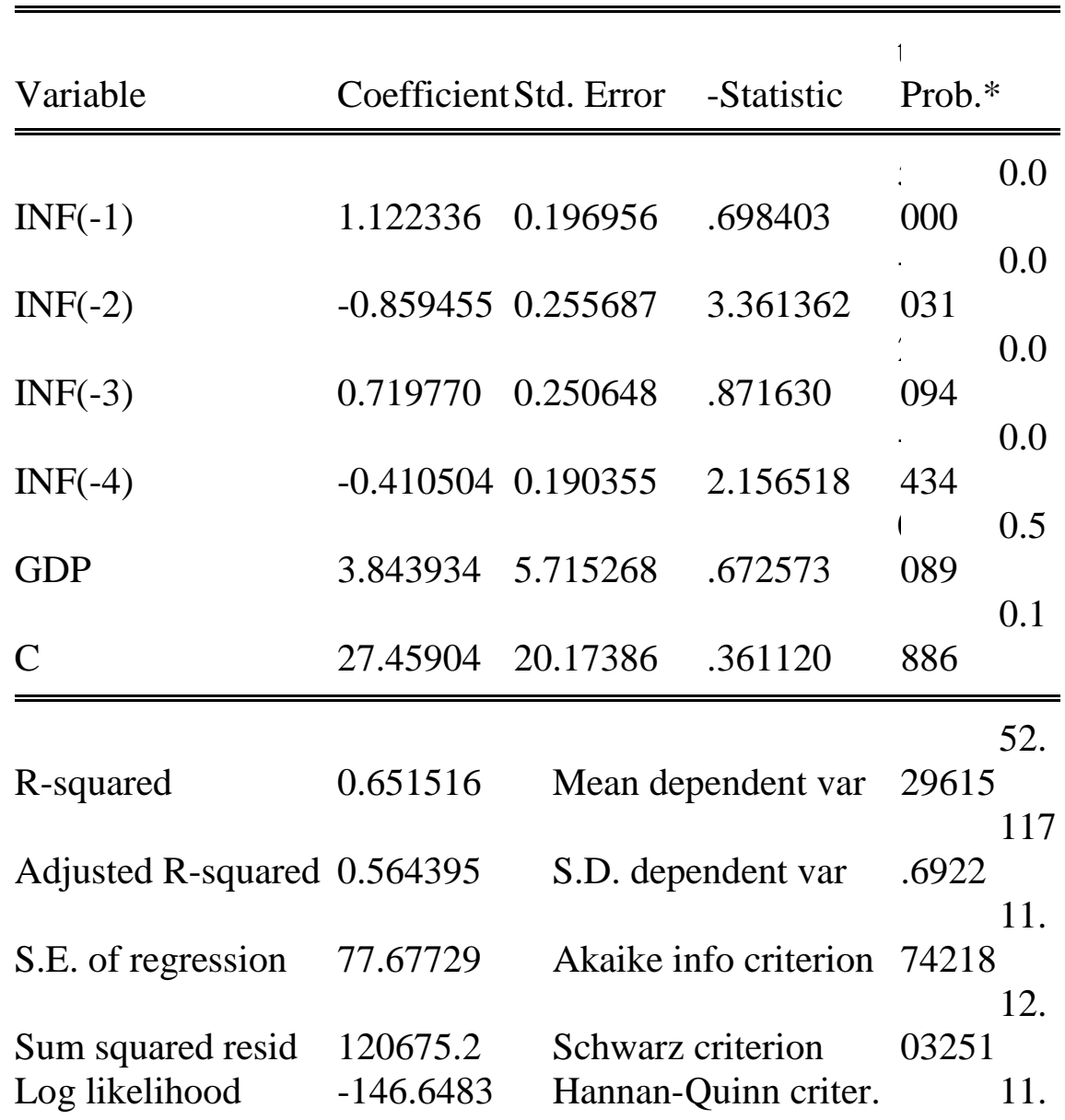

TANMIYAT AL-RAFIDAIN (P-ISSN: 1609-591X; E-ISSN: 2664-276X) تنمية الرافدين 
82578

1.1

F-statistic $\quad 7.478295 \quad$ Durbin-Watson stat 69107

Prob(F-statistic) $\quad 0.000423$

*Note: p-values and any subsequent tests do not account for model selection.

EViews 10 المصدر: من إعداد الباحثين باستخدام برنامج

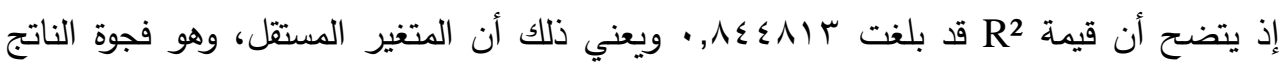

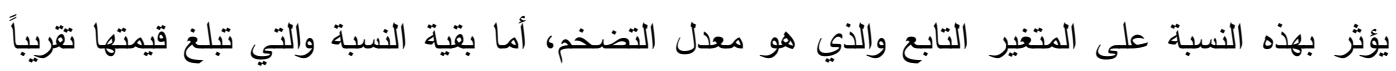

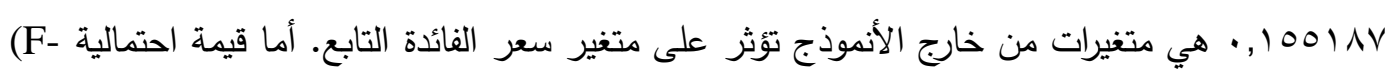

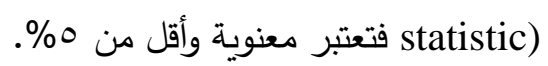

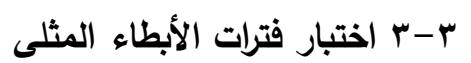

Akaike يتم من خلال هذا المعيار إجراء اختبار فترات الابطاء المثلى بالاعتماد على معيار Information Criteria (AIC) القيم الصغرى هي القيم المثلى عند اختيار الأنموذج، من هنا سيكون الأنموذج (4,0) هو الأنموذج

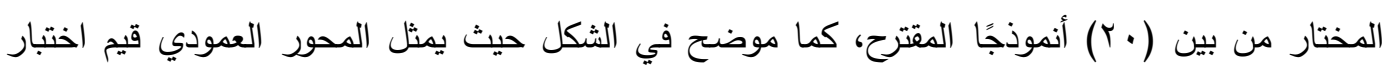

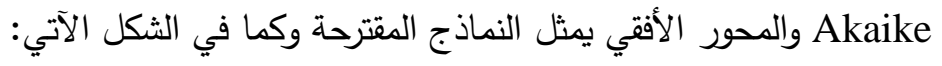

الثكل ؛ : تحديد الأنموذج القياسي الأمثل 
Akaike Information Criteria

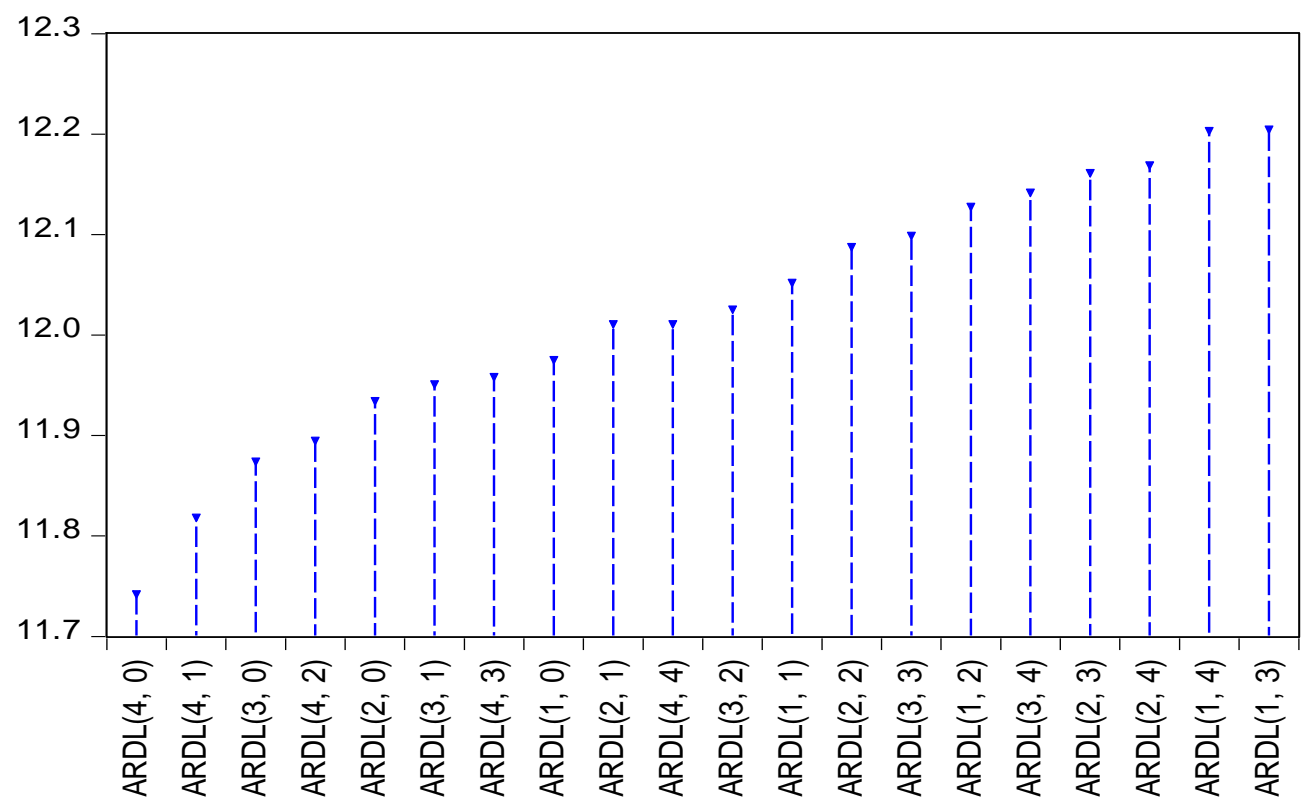

EViews 10 المصدر: من عمل الباحثين بالاعتماد على برنامج

rounds Test r- rاختبار التكامل المشترك (اختبار الحدود) يتم من خلال اختبار الحدود الكثف عن درجة وجود التكامل المشترك بين متغيرات الأنموذج وكما مبين في الجدول (0) الآتي:

الجدول ه: نتائج اختبار التكامل المشترك (اختبار الدود)

F-Statistics $=4.873288$

\begin{tabular}{c|c|c}
\hline Signif. & Lower Approach I(0) & Upper Approach I(1) \\
\hline $10 \%$ & 3.02 & 3.51 \\
\hline $5 \%$ & 3.62 & 4.16 \\
\hline $2.5 \%$ & 4.18 & 4.79 \\
\hline $1 \%$ & 4.94 & 5.58 \\
\hline
\end{tabular}

EViews المصدر: من إعداد الباحثين باستخدام برنامج 10

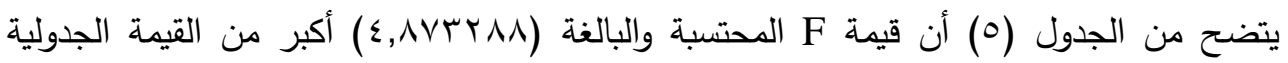
للحدود الصغرى والعظمى الجدولية عند مستوى (•(\%) عند التخلف الزمني (ع،ء) كأفضل فترة إبطاء زمنياً، وهذا يعني رفض فرضية العدم القائلة بعدم وجود تكامل مشترك H0: Lai=0 بين متغيرات الأنموذج 


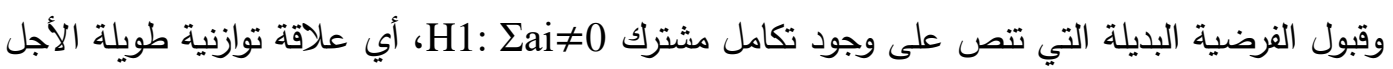

$$
\text { بين متغيرات الأنموذج. }
$$

r-ه تقدير معلمات الأنموذج في الأجلين الطويل والقصير ومعلمة تصحيح الخطأ

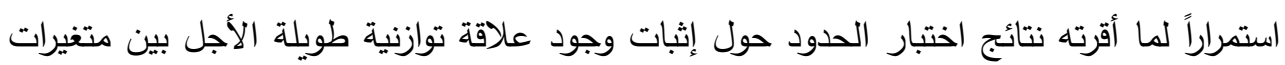
الأنموذج، لا بد من تقدير معلمات الأنموذج (ARDL) في الأجلين الطويل والقصير ومعلمة تصحيح

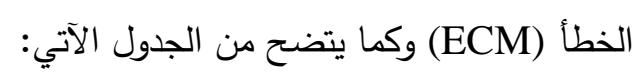

الجدول 1: نتائج معلمات الأجلين الطويل والقصير ومعلمة تصديح الاتئ الخطأ

ARDL Long Run Form and Bounds Test

Dependent Variable: D(INF)

Selected Model: ARDL $(4,0)$

Case 2: Restricted Constant and No Trend

Date: 08/03/20 Time: 14:16

Sample: 19892018

\begin{tabular}{|c|c|c|c|c|}
\hline \multicolumn{5}{|c|}{ Conditional Error Correction Regression } \\
\hline Variable & Coefficient & Std. Error & t-Statistic & Prob. \\
\hline $\mathrm{C}$ & 27.45904 & 20.17386 & 1.361120 & 0.1886 \\
\hline $\operatorname{INF}(-1)^{*}$ & -0.427853 & 0.181430 & -2.358233 & 0.0287 \\
\hline $\mathrm{GDP}^{* *}$ & 3.843934 & 5.715268 & 0.672573 & 0.5089 \\
\hline $\mathrm{D}(\mathrm{INF}(-1))$ & 0.550189 & 0.219112 & 2.511000 & 0.0207 \\
\hline $\mathrm{D}(\mathrm{INF}(-2))$ & -0.309266 & 0.177422 & -1.743112 & 0.0967 \\
\hline $\mathrm{D}(\operatorname{INF}(-3))$ & 0.410504 & 0.190355 & 2.156518 & 0.0434 \\
\hline
\end{tabular}

Included observations: 26

* p-value incompatible with t-Bounds distribution.

** Variable interpreted as $\mathrm{Z}=\mathrm{Z}(-1)+\mathrm{D}(\mathrm{Z})$.

Levels Equation

\begin{tabular}{|c|c|c|c|c|}
\hline Variable & Coefficient & Std. Error & t-Statistic & Prob. \\
\hline GDP & 8.984235 & 13.12304 & 0.684615 & 0.5014 \\
\hline $\mathrm{C}$ & 64.17865 & 37.67432 & 1.703512 & 0.1040 \\
\hline
\end{tabular}

Case 2: Restricted Constant and No Trend

ARDL Error Correction Regression

Dependent Variable: D(INF)

Selected Model: ARDL $(4,0)$

TANMIYAT AL-RAFIDAIN (P-ISSN: 1609-591X; E-ISSN: 2664-276X) تنمية الرافدين 
Case 2: Restricted Constant and No Trend

Date: 08/03/20 Time: 14:17

Sample: 19892018

Included observations: 26

ECM Regression

Case 2: Restricted Constant and No Trend

\begin{tabular}{|c|c|c|c|c|}
\hline Variable & Coefficient & Std. Error & t-Statistic & Prob. \\
\hline $\mathrm{D}(\mathrm{INF}(-1))$ & 0.550189 & 0.207579 & 2.650511 & 0.0153 \\
\hline $\mathrm{D}(\mathrm{INF}(-2))$ & -0.309266 & 0.167813 & -1.842923 & 0.0802 \\
\hline $\mathrm{D}(\mathrm{INF}(-3))$ & 0.410504 & 0.181133 & 2.266315 & 0.0347 \\
\hline CointEq(-1)* & -0.427853 & 0.172082 & -2.486331 & 0.0219 \\
\hline R-squared & 0.477294 & \multirow{6}{*}{\multicolumn{2}{|c|}{$\begin{array}{l}\text { Mean dependent var } \\
\text { S.D. dependent var } \\
\text { Akaike info criterion } \\
\text { Schwarz criterion } \\
\text { Hannan-Quinn criter. }\end{array}$}} & -3.203846 \\
\hline Adjusted R-squared & 0.406016 & & & 96.09710 \\
\hline S.E. of regression & 74.06239 & & & 11.58833 \\
\hline Sum squared resid & 120675.2 & & & 11.78188 \\
\hline Log likelihood & -146.6483 & & & 11.64407 \\
\hline Durbin-Watson stat & 1.169107 & & & \\
\hline
\end{tabular}

* p-value incompatible with t-Bounds distribution.

EViews 10 المصدر: من إعداد الباحثين بالاعتماد على برنامج

يتبين من الجدول (T) بأنه يوجد هناك علاقة طويلة الأجل بين فجوة الناتج ومعدل التضخم،

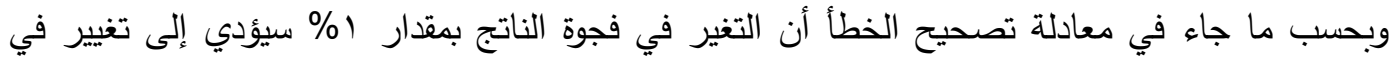

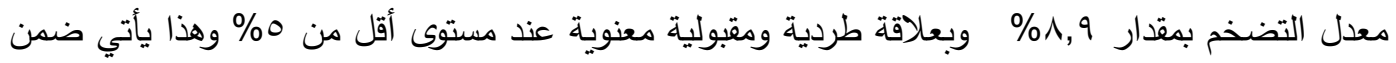
ما تتص عليه النظرية الاقتصادية، أما بالنسبة للعلاقة قصيرة الأجل فقد ظهرت العلاقة طردية أيضاً بين

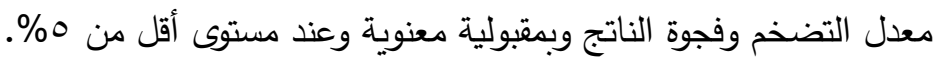

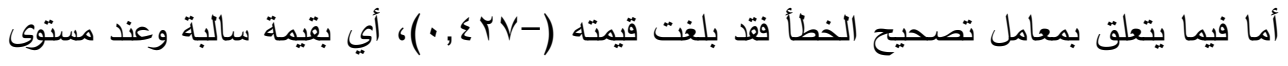
معنوية أقل من ٪\%، أي أن الانحرافات التي تحصل في الأجل القصير يمكن تصحيحها خلال ( من الزمن، وعلية يتأكد وجود علاقة توازنية طويلة الأجل بين متغيرات الأنموذج ورفض فئ فرضية الاجن العدم والقبول بالفرضية البديلة التي تتص على وجود علاقة توازنية طويلة الأجل بين المتغيرات في الأنموذج. r- إ إجراء الاختبارات التشخيصية

لغرض التأكد من صحة جودة أداء الأنموذج، يمكن ذلك من خلال إجراء مجموعة من الاختبارات

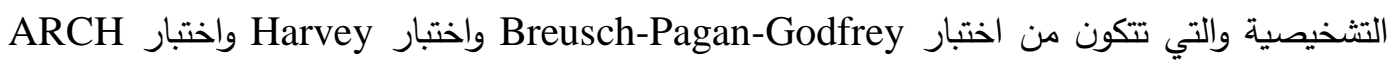
وظهرت النتائج كما مبين في الملحق (1). وبينت جميع الاختبارات التي اعتمدت على قيمة (Prob. F) و 
أنها غير معنوية عند مستوى ٪\% (Prob. Chi-Square) ثبات تجانس التباين.

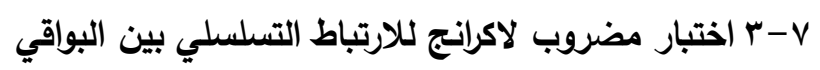
يتم من خلال اختبار مضروب لاكرانج للارتباط التسلسلي بين البواقي الكثف عن الإنف لإنباط

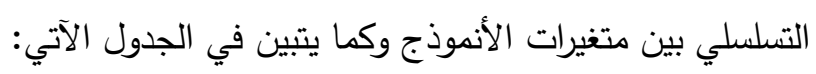
الجدول V: نتائج اختبار مضروب لاكرانج للارتباط التسلسلي بين لانين البواقي

Breusch-Godfrey Serial Correlation LM Test:

\begin{tabular}{llcc}
\hline \hline F-statistic & 2.665280 & Prob. F(2,18) & 0.0969 \\
Obs*R-squared & 5.940474 & Prob. Chi-Square(2) & 0.0513 \\
\hline \hline
\end{tabular}

EViews 10 المصدر: من إعداد الباحثين بالاعتماد على برنامج

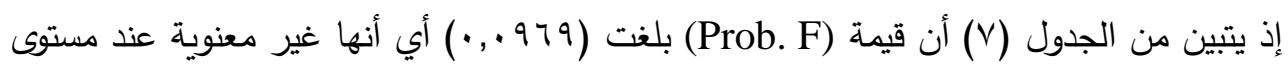

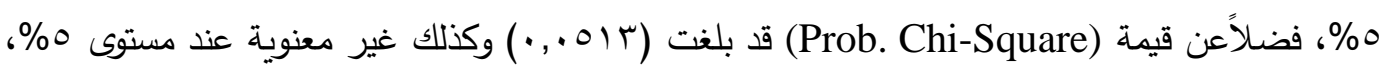
أي أن الأنموذج لا يعاني من مشكلة الارتباط التسلسلي، وبالتالي نرفض الفرضية البديلة التي تحتم وجود

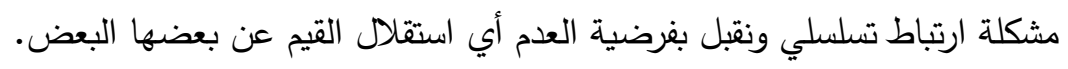
ب-1 اختبار توزيع الأخطاء العشوائية

أظهرت نتائج اختبار توزيع الأخطاء العشوائية أن متغيرات البواقي (Residuals) لا لأتوزع توزيهاً طبيعياً، حيث هناك انحراف في قيم البواقي عن وسطها الحسابي وبقيم غير معنوية، وكما مبين في الثكل 


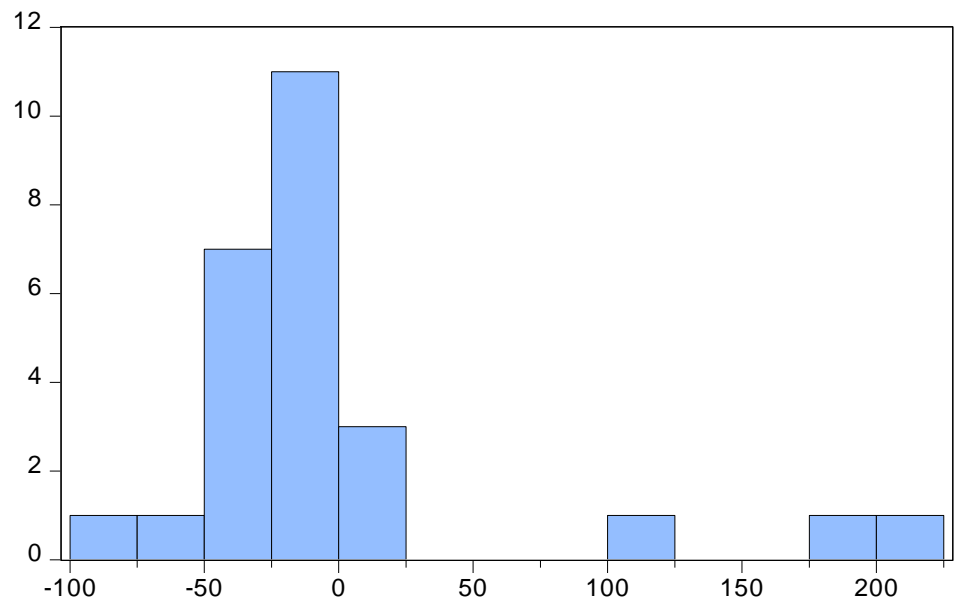

Series: Residuals

Sample 19932018

Observations 26

Mean $\quad-1.36 \mathrm{e}-14$

Median $\quad-17.38095$

Maximum 214.0224

Minimum $\quad-98.72572$

Std. Dev. $\quad 69.47668$

Skewness $\quad 2.010572$

Kurtosis $\quad 6.567276$

Jarque-Bera $\quad 31.30298$

Probability $\quad 0.000000$

EViews المصدر: من إعداد الباحثين بالاعتماد على برنامج 10

ץ-9 اختبار الاستقرارية الهيكلية لمعلمات الأنموذج

لغرض التأكد من عدم احتواء البيانات الداخلة في الأنموذج أي تغيرات هيكلية فيها ومدى استقرار الأنيات

وانسجام المعاملات طويلة الأجل مع التقديرات لمعلمات الأجل القصير، لذلك تظهر نتائج اختبار التهري

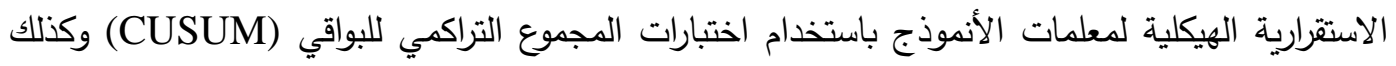

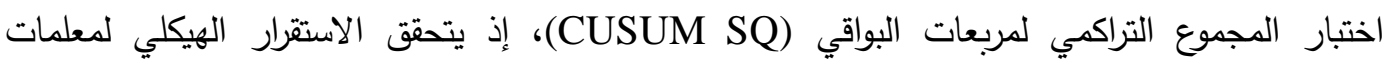

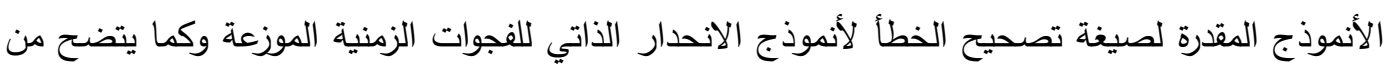

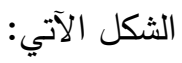


تقدير العلاقة بين فجوة الناتج والتضخم ................

عقراوي والجويجاتي

الثكل ج": الاستقرارية الهيكلية لمعلمات الأنموذج
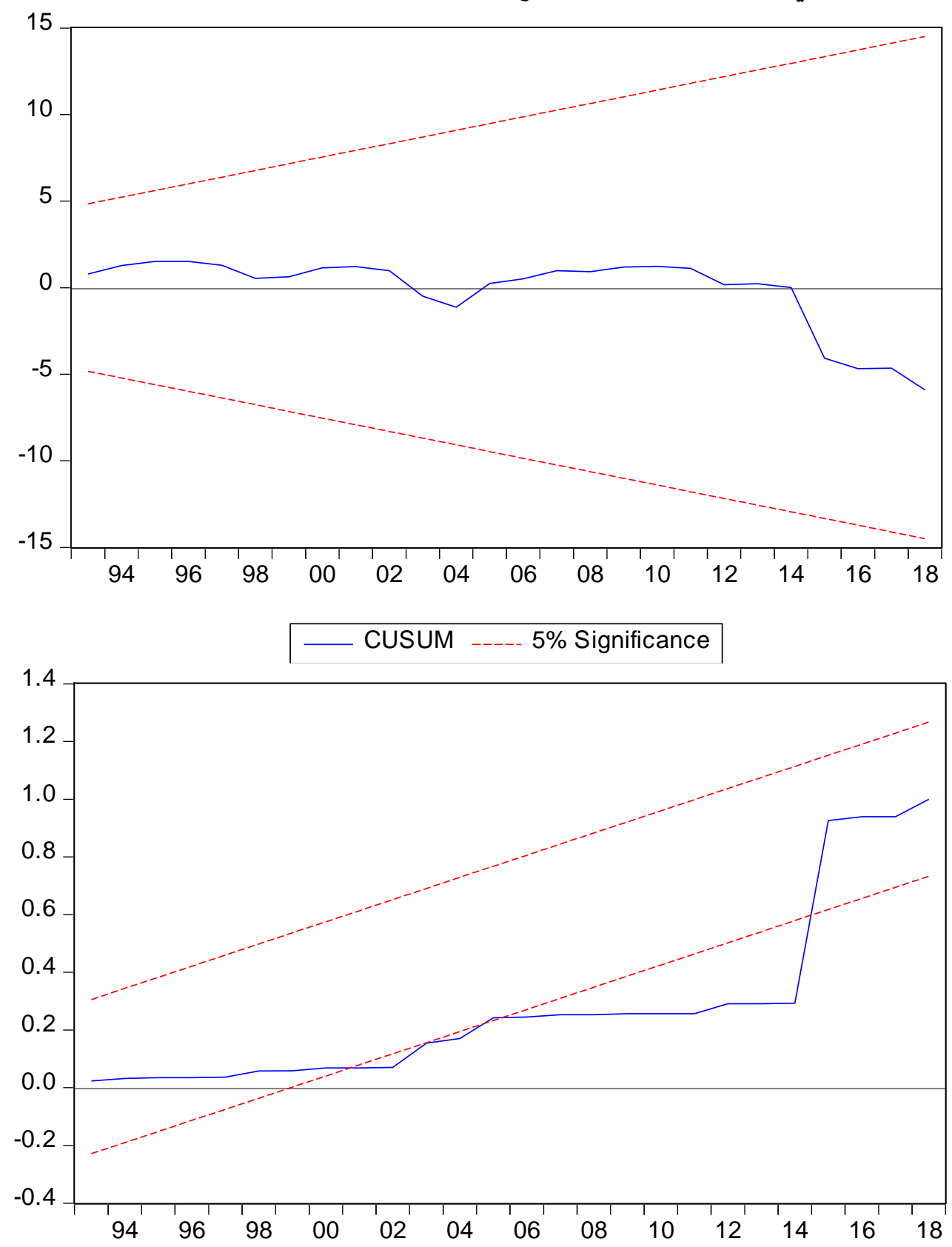

_- CUSUM of Squares ----- 5\% Significance

EViews المصدر: من إعداد الباحثين بالاعتماد على برنامج 10

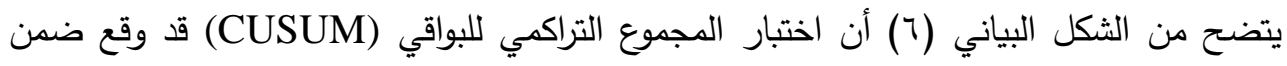

الحدود الحرجة عند مستوى معنوية 0\%، مما يعني أن المعاملات المقدرة لأنموذج تصحيح الخطأ غير لئر

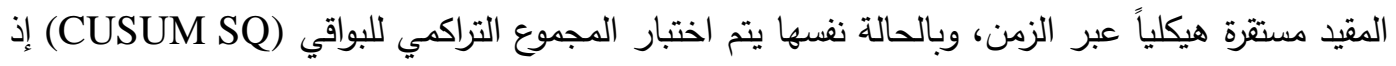

TANMIYAT AL-RAFIDAIN (P-ISSN: 1609-591X; E-ISSN: 2664-276X) تنمية الرافدين 
وقع أيضاً إلا في سنوات معينة خارج الحدود الحرجة عند مستوى معنوية ٪\%، لذلك يشير الثكلان إلى إلى وجود نوع من الاستقرار والانسجام بين معلمات الأجل الطويل ومعلمات الأجل القصير للكأنموذج. r-1 اختبار الأداء التنبؤي لأنموذج تصحيح الخطأ لغرض التأكد من أن الدالة تتمتع بقدرة عالية على التتبؤ خلاءل الفتواء الفترة الزمنية للدراسة، يستخدم مجموعة من المعايير لغرض قياس الأداء التنبؤي للنماذج القياسية منها: - معامل عدم التساوي لثايل (T): ويعتبر من أهم المعايير المستخدمة في قياس واختبار القدرة التتبؤية للنماذج القياسية والتأكد من دقة عملية التتبؤ. - معيار نسبة عدم التساوي، ويتكون من ثلاث نسب هي نسبة التحيز (BP)، نسبة التباين (VP) ونسبة التغاير (CP).

الثكل V: الأداء التنبؤي لأنموذج تصحيح الخطأ

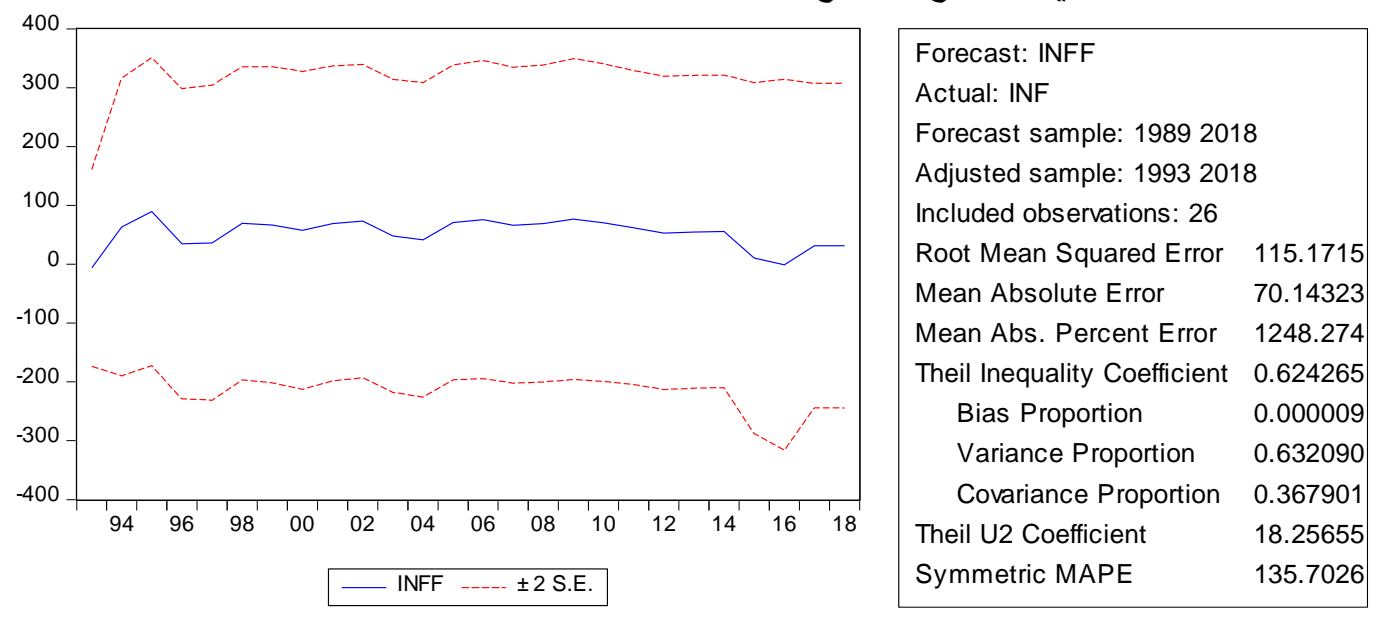

EViews المصدر: من إعداد الباحثين بالاعتماد على برنامج 10

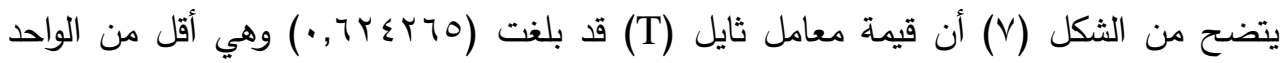

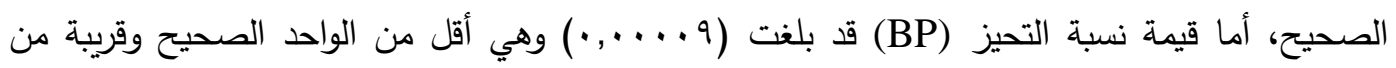

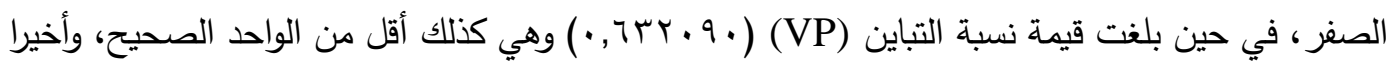

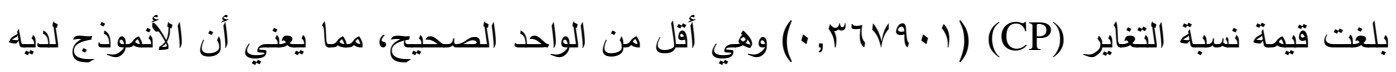
القدرة العالية على التتبؤ في الددة الزمنية للدراسة، ويمكن الاعتماد عليه في التحليل وتقييم السياسات والتتبؤ بها في المستقبل ولغرض اتخاذ القرارات الاقتصادية الصحيحة للوصول إلى الأهداف المطلوبة. 


\section{ع - الاستنتاجات والتوصيات

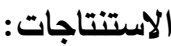

1- يؤدي تقدير فجوة الناتج دورًا كبيرًا في إعطاء المعلومات عن حالة الاقتصاد، حيث يهتم واضعو السياسة النقدية في عملية تقدير فجوة الناتج في وضع السياسة المطلوبة التي تعمل على إيجاد الحلول للتقلبات الحاصلة في الاقتصاد الحد من تغيراتها. ץ- يعتبر مرشح كالمن متعدد المتغيرات من الطرائق الإحصائية الاقتصادية في تقدير فجوة الناتج، لأنه

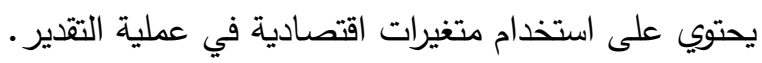
r- شهد النشاط الاقتصادي العراقي تقلبات كثيرة نتيجة للتغيرات الاقتصادية التي عاشها البلد، ابتداءً من فئن

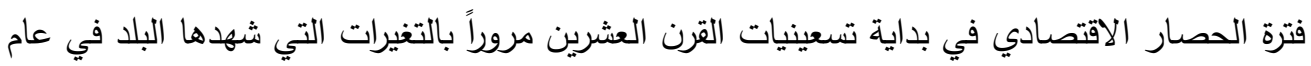

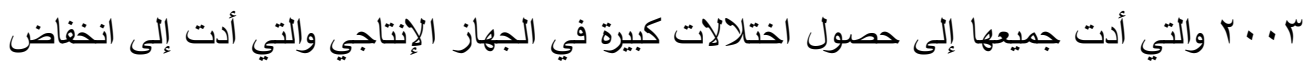
الناتج المحلي الإجمالي وبالتالي التأثير على فجوة الناتج. ع- على مستوى معدل التضخم أيضاً شهد تقلبات كبيرة في معدلاته نتيجة لمتغيرات اقتصادية كبيرة منها انخفاض الناتج المحلي الإجمالي وكذلك عملية تغطية الانفاق المالي عبر الإصدار النقدي الذي لدئي

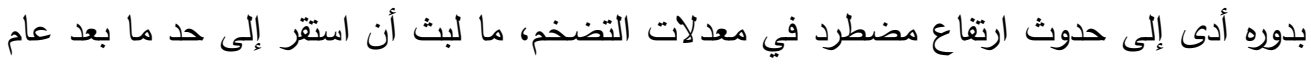

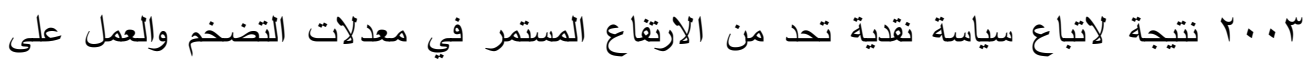
استقرار معدلاته.

0- تبين من خلال اختبار الاستقرارية (جذر الوحدة) أن الأنموذج القياسي الأنسب في تحديد شكل

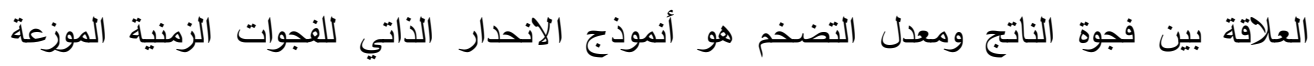

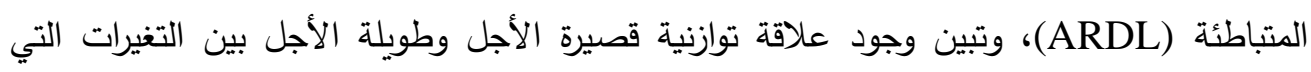
تحصل في فجوة الناتج كمتغير مستقل ومعدل التضخم كمتغير تابع. التوصيات 1- ضرورة تقدير فجوة الناتج وحسابها والعمل على استقرار معدلاتها بحيث يتساوى الناتج الفعلي مع الناتج المستهدف للدور الكبير الذي يمكن أن تؤديه في تحقيق الاستقرار في معدل التضخد. r- ضرورة الحد من الارتفاع والتغييرات الكبيرة التي تحصل في معدل التضخم من خلال الحد من تأثير المتغيرات الاقتصادية التي تؤثر على معدلات التضخم والتقليل من تقلباتها. r- يوصي الباحثان بضرورة اختيار الأنموذج القياسي الأمثل بناءً على الاختبارات الرياضية والقياسية

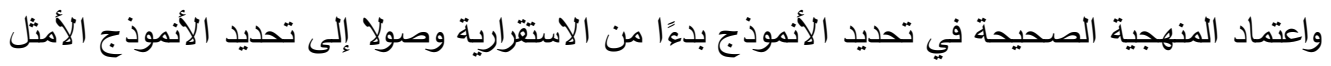

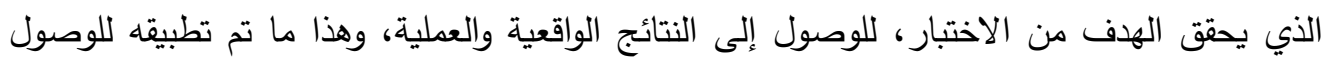
Refrence

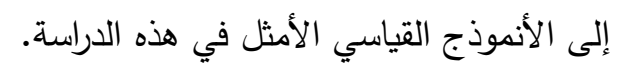


Almari, A. \& Almisbah, A. (2016)," Estimating GDP Gap Saudi in the period 1970-2012 Applied study by using Hodrick-Prescott filter and MKF", Center for Arab unity studies, Economic Research Journal, Issue 74-75.

Apel, Michael, Hansen, Jan, Lindberg, Hans, 1996, potential output and output gap, Sverige's riksbank, quarterly review, 3.

Central Bnk of Iraq, Annual Statistical Bulletin, Different Years.

Hodrick, Robert J, Prescott, Edward C, 1997, postwar US business cycles: an empirical investigation, journal of money, credit and banking.

Jahan, S. Mahmood, S. (2013)" What the meant by GDP Gap", (IMF), Journal of Finance and Development, Vol. 50, Issue 3.

Kadhim, S. Mohammed, S. \& Khlaf, H. (2019)," Change in mpney supply and its impact on inflation and output in Iraq for the period 1990-2016", Almamoon college Journal, Issue 34, pp 114-138.

Kiley, Michael, 2013, output gaps, journal of macroeconomics, Elsevier, 37.

Kramer, J. \& Farrell, G. (2014) "the reliability of south Africa real- time output gap estimates", ERSA working paper 428.

Ministry of Planning, Central Statistical Organization, Different Years.

Najmaldin, K. Hameed, A. \& Ahmed, T. (2012),'Economic inflation and analysis of the casual relationship between inflation and money supply in Iraq for the period 1985-2008" Alkut Journal foe the economics and the management science, Vol. 1, Issue 6, pp. 335-361.

Salih, M. (2015), "Money supply and its impact on inflation an analytical study on the Iraqi economy", ALdananeer Journal, Issue 7, pp. 257-279.

Szulczyk, Kenneth R. (2014), Money Banking and International Finance, Second Edition Kenneth R. Szulczyk. 